\title{
WETTING CRITERIA FOR THE APPLICABILITY OF MEMBRANE DISTILLATION*
}

\author{
A.C.M. FRANKEN, J.A.M. NOLTEN, M.H.V. MULDER, D. BARGEMAN and C A. \\ SMOLDERS \\ Twente Unversity, Department of Chemical Technology, P O Box 217, 7500 AE Enschede \\ (The Netherlands)
}

(Recerved November 17, 1986; accepted in revised form April 7, 1987)

\section{Summary}

Membrane distillation can only be applied on hquid mixtures which do not wet a microporous hydrophobic membrane. Solutions of inorganic material in water have such high values of surface tension $\left(\gamma_{\mathrm{L}} \geqslant 72 \times 10^{-3} \mathrm{~N} / \mathrm{m}\right)$ that the non-wetting condition is fulfilled for a number of hydrophobic membranes. As soon as organic solutes are present in the solution, the surface tension $; \mathrm{i}$ will be lowered, and if the concentration of organic material becomes too high, wetting of the membrane will occur. By means of theoretical considerations a critical solute concentration or surface tension at which a homogeneous smooth material will be wetted $\left(\theta<90^{\circ}\right)$ can be calculated. For (micro) porous membranes no such theoretical relation can be derived. Therefore, a simple experimental method is described to measure the maxinum allowable concentration for a (micro) porous membrane. On the basis of these measurements, the maximum allowable concentration under process conditions can be determined

\section{Introduction}

Membrane distillation is a distillation process which makes use of the pores of a microporous non-wettable membrane as the vapour phase. In this process two aqueous liquids with different temperatures are separated by a hydrophobic microporous membrane. The vapour pressure difference $A P_{\mathrm{v}}$ across the membrane, resulting from the temperature difference $A T$, causes vapour molecules to be transported through the pores of the membrane from the warm side (feed) to the cold side (permeate).

The advantages of membrane distillation are that the distillation process takes place at moderate temperature and that a relatively low temperature difference between the two liquids contacting the microporous hydrophobic membrane gives relatively high fluxes. Because entrainment of dissolved particles is avoided, a permeate with a high purity is obtained.

\footnotetext{
*Paper presented at the Workshop on Membrane Distillation, Rome, Italy, May 5, 1986.
} 
However, membrane distillation is only possible if the restrictive condition is fulfilled that the pores of the membrane are not filled with liquid. Hence the wetting power of the liquids should be low. Water and solutions of inorganic substances in water have such high values of surface tension $\left(\gamma_{\mathrm{L}} \geqslant 72 \times 10^{-3}\right.$ $\mathrm{N} / \mathrm{m}$ ) that for a number of hydrophobic microporous membranes with pores in the range of $1 \mu \mathrm{m}$ or less ( such as polypropylene (PP), polyvinylidene fluoride (PVDF) and polytetrafluoroethylene (PTFE, Teflon)) this non-wetting condition is guaranteed. Therefore, important applications of membrane distillation can be seen in the field of water purification and in concentration of product solutions or waste water solutions $[1,2]$.

When organic solutes are present in an aqueous solution, the surface tension $\gamma_{L}$ will decrease rapidly. If the concentration of organic material does not exceed a certain critical value (so that the liquid on both sides of the membrane does not wet the membrane), the membrane distillation process can still be used. On the other hand, if the concentration of organic material exceeds this critical value, the microporous membrane will be filled with liquid. In this case membrane distillation is no longer possible.

The aim of this investigation is to find out which concentration of organic material in water is allowed before the liquid will penetrate into the membrane.

\section{Background}

The value of the contact angle $\theta$ of a liquid droplet on an ideal smooth homogeneous surface is described by Young's equation:

$\gamma_{\mathrm{L}} \cos \theta=\gamma_{\mathrm{S}}-\gamma_{\mathrm{SL}}$

A droplet of water on a hydrophobic surface (e.g. PP, PVDF or PTFE) will give a contact angle which is larger than $90^{\circ}$. If surface active agents (or in general: organic materials ) are dissolved in water, the surface tension of the liquid will decrease. As a consequence, the contact angle $\theta$ will decrease, and if $\theta$ becomes smaller than $90^{\circ}$ the liquid will wet the solid surface. In case the material is non-poruus the contact angle will have a value between $0^{\circ}$ and $90^{\circ}$ On the other hand, if the material is porous (which is the case for membranes used in the membrane distillation process) it is possible that the droplet will penetrate into the pores of the material.

Lucassen-Reynders [3] stated that any of the interfacial tensions in eqn. (1) can be affected by surfactant adsorption by virture of Gibb's law:

$\frac{\mathrm{d} \gamma_{\iota}}{\mathrm{d}\left(\ln a_{\imath}\right)}=-R T \Gamma_{\iota} \quad$ with $\imath=\mathrm{L}, \mathrm{S}, \mathrm{SL}$

$a_{i}$ being the activity of the surfactant and $\Gamma_{l}$ its surface excess at any interface. Adsorption of surfactants can only influence the contact angle if they affect the ratio $\left(\gamma_{\mathrm{S}}-\gamma_{\mathrm{SL}}\right) / \gamma_{\mathrm{L}}$. Changes in contact angle can be shown conveniently 


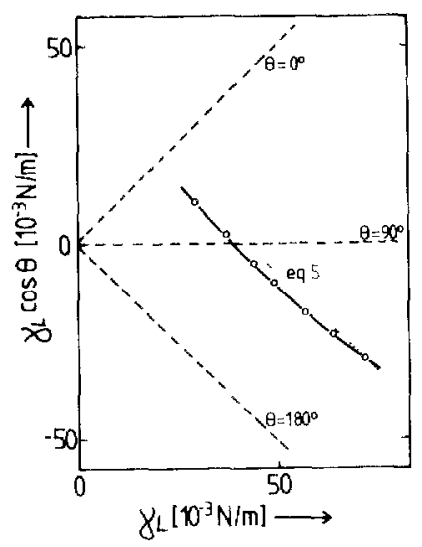

Fig. $1 \gamma_{L} \cos \theta$ as a function of $\gamma_{L}$ for ethanol-water mixtures on a homogeneous PTFE surface, the dotted line is calculated according to eqn. (5)

by plotting $\left(\gamma_{S}-\gamma_{\mathrm{SL}}\right)$ as a function of $\gamma_{\mathrm{L}}$. Combination of eqns. (1) and (2) then yields the following expression:

$\frac{\mathrm{d}\left(\gamma_{\mathrm{L}} \cos \theta\right)}{\mathrm{d} \gamma_{\mathrm{L}}}=\frac{\Gamma_{\mathrm{S}}-\Gamma_{\mathrm{SL}}}{\Gamma_{\mathrm{L}}}$

For low energy surfaces (such as PP, PVDF or PTFE) it is expected that hardly any interaction exists between the surface active agents and the surface, in which case $\Gamma_{\mathrm{SL}} \approx \Gamma_{\mathrm{L}}$. It is also expected that $\Gamma_{\mathrm{S}} \ll \Gamma_{\mathrm{SL}}[4]$, and consequently the slope of the curve as represented by eqn. (3) will be

$\frac{\mathrm{d}\left(\gamma_{\mathrm{L}} \cos \theta\right)}{\mathrm{d} \gamma_{\mathrm{L}}} \approx-1$

Bargeman and Van Voorst Vader [4] indeed found this relation for solutions of sodium decane-1-sulphonate and sodium dodecane sulphate in water on nonpolar solids such as paraffin wax and PTFE. For conditions as assumed up till now the influence of surfactants on the contact angle on non-polar solids can be described by

$\gamma_{L} \cos \theta=-\gamma_{L}+C$

This linear equation with a slope equal to -1 has the advantage that only one contact angle measurement needs to be performed: the measurement of the contact angle of pure water on the solid material. If the surface tension of the solution as a function of the composition is known, the value of $\gamma_{\mathrm{L}}$ and therefore the value of the contact angle $\theta$ can be calculated for each solution composition. This situation is represented by the dotted line in Fig. 1.

In Figure 1 the value of $\gamma_{\mathrm{L}}^{90}$ is given by the intercept on the abscissa. $\gamma_{\mathrm{L}}^{90}$ is the surface tension of a liquid mixture which has a contact angle of $90^{\circ}$ when 
brought in contact with a homogeneous smooth solid material. In the membrane distillation process the value of $\gamma_{\mathrm{L}}^{90}$ is very important. If the surface ten sion of a liquid is lower than $\gamma_{\mathbf{L}}^{90}$ it may be possible for the liquid to penetrate into the pores of the porous material spontaneously.

The surface tension of a liquid which is on the verge of penetration into the pores of a (micro) porous membrane is defined as $\gamma^{\mathrm{p}}$ ( $\mathrm{p}$ stands for penetration). As far as membrane distillation is concerned the value of $\gamma \mathrm{p}$ is even more important than the value of $\gamma_{\mathrm{L}}^{90}$.

Although the above described method for the determination of $\gamma_{\mathrm{L}}^{90}$ seems to be promising, it cannot be used as an accurate determination of $\gamma \mathrm{l}$. This is mainly due to three effects:

1. The experimental error for points on a line with a certain inaccuracy in its slope drawn through one experimental point with a certain inherent error increases with the distance to that point.

2. The use of eqn. (5) is limited to surface active agents of a certain molecular structure (rather long chain amphipolar molecules, so called surfactants).

3. The value of $\gamma_{\mathrm{L}}^{90}$ does not always coincide with the value of $\gamma_{\mathrm{L}}^{\mathrm{p}}$ for porous media.

Re 1 . Although contact angle measurements can be made very accurately some deviations are inevitable. Mostly a deviation of about $1^{\circ}$ is given. In literature the differences between the measured contact angles may differ considerably. For example, for a droplet of water on a Teflon surface, contact angles varying from $108^{\circ}$ to $115^{\circ}$ have been reported [5-8]. This effect is mainly due to the use of different specimens of Teflon. Another source of error is the inaccuracy in the slope of the curve: the slope is not exactly -1. For different series of measurements Bargeman and Van Voorst Vader [4] found that the slope varied between -0.96 and -1.02 . Therefore, the effect of an experimental error in the contact angle is reinforced by the uncertainty in the tangent of the slope. For example, if for a droplet of water on Teflon a contact angle $\theta=114^{\circ} \pm 1^{\circ}$ is found and the tangent of the slope is uncertain within $2 \%$, then the extreme values for $\gamma_{\mathrm{L}}^{90}$ are $40 \times 10^{-3} \mathrm{~N} / \mathrm{m}$ and $46 \times 10^{-3} \mathrm{~N} / \mathrm{m}$, respectively

Re 2. Equation (5) is used to describe the influence of surfactants on the contact angle of non-polar solids, and a good agreement with experimental results is found [4]. On the other hand, this relation cannot be used in general for mixtures of water and low molecular weight organic components, such as alcohols (methanol, ethanol) or carboxylic acids (formic acid, acetic acid). In these cases a linear behaviour as described by eqn. (5) is not always found. As an example the curve for ethanol/water mixtures on a homogeneous PTFE surface is given in Fig. 1 (the experimental results were obtained from Bernett and Zisman [6]).

$\operatorname{Re} 3$. The difference between $\gamma_{\mathrm{L}}^{90}$ and $\gamma_{\mathrm{L}}^{\mathrm{p}}$ will be demonstrated and explained on the bases of the results of our experiments. 
The restrictions mentioned above do not permit an accurate determination of $\gamma_{\mathrm{L}}^{90}$ on the basis of the measurement of the contact angle of a droplet of water on a solid. Furthermore it is doubtful whether the value for $\gamma_{\mathrm{L}}^{90}$ is identical to that for $\gamma \mathrm{L}$.

From the above considerations it can be concluded that the value of $\gamma \mathrm{L}$ has to be determined experimentally for the microporous membrane used.

\section{Method of investigation}

\section{Determination of $\gamma \mathrm{P}$}

The value of $\gamma^{\mathrm{p}}$ is determined by the 'penetrating drop method'. In this method a droplet is brought in contact with the (micro) porous membrane. By trials with narrowing series of solution compositions the composition of a liquid mixture is determined at which the liquid is on the verge of penetration into the membrane. The amount of organic material in water at this composition is called the 'maximum allowable concentration' and $\nu^{p}$ is defined as the value of the surface tension belonging to this composition. The advantages of the penetrating drop method are:

- the value of $\gamma \mathrm{p}$ can be measured directly,

- the measurements can be carried out on the membrane material itself,

- the method is experimentally simple and requires no special equipment,

- the measurements can be carried out very quickly,

- the method has a high accuracy (compared to the contact angle measurement).

The accuracy of the penetrating drop method can be illustrated by the following example. A droplet will have a height of less than $5 \mathrm{~mm}$ and therefore will exert a gravitational force of about $50 \mathrm{~N} / \mathrm{m}^{2}$, which might form an inaccuracy in determining $\gamma \mathrm{L}$. Suppose the maximum pore size of the membrane material is smaller than $10 \mu \mathrm{m}$. According to the Laplace equation,

$\Delta P=-\frac{2 B \gamma_{\mathrm{L}} \cos \theta}{r_{\max }}$

the value of $\gamma_{\mathrm{L}} \cos \theta$ can be measured with an accuracy of less than $0.25 \times 10^{-3}$ $\mathrm{N} / \mathrm{m}$. In the above equation $B$ is a pore geometry coefficient, being 1 for cylindrical pores. Using eqn. (4) for an estimation of the uncertainty in $\gamma^{p}$, in this case $\Delta \gamma \mathrm{p}$, it follows that $\Delta \gamma \mathrm{p}<0.25 \times 10^{-3} \mathrm{~N} / \mathrm{m}$.

Calculation of $\gamma \mathrm{P}$ under process conditions $\left(\gamma_{\mathrm{L}, \mathrm{pc}}^{\mathrm{p}}\right)$

The preceding explanation of $\gamma \mathrm{p}$ and its determination is only partly appli- 
cable to hydrophobic microporous membranes under process conditions. As far as intrinsic membrane properties are concerned the preceding discussion remains unaltered.

Nevertheless, it can be easily understood that a liquid mixture with a composition just beneath the maximum allowable concentration can give problems in the membrane distillation process. In practical applications the pressure, exerted by the liquid on the membrane, will be higher than zero as a result of, among other things, pumping pressure. If in that case the liquid has a surface tension $\gamma_{\mathrm{L}}$ which is only slightly higher than $\gamma_{\mathrm{P}}^{\mathrm{p}}$, it is possible that the liquid penetrates into the microporous membrane.

Therefore, $\gamma_{\mathrm{L}, \mathrm{pc}}^{\mathrm{p}}$ is introduced. The surface tension $\gamma_{\mathrm{L}, \mathrm{pc}}^{\mathrm{p}}$ is defined as the 'minimum allowable surface tension under process conditions'. The concentration corresponding to $\gamma^{\mathrm{R}}, \mathrm{pc}$ is called 'maximum allowable concentration under process conditions'.

The relation between the applied pressure and the surface tension is given by the Laplace equation (eqn. 6). If the value of the maximum pore size is known and an estimation is made of the applied pressure, then the value of $\gamma_{L}$. $\cos \theta$ can be calculated. If the curve for $\gamma_{L} \cos \theta$ as a function of the concentration is known, then the value of $\gamma_{\mathrm{l}, \mathrm{pc}}^{\mathrm{c}}$ can be obtained graphically. Determination of $\gamma_{\mathrm{L}, \mathrm{pc}}$ in this way makes use of the assumption that $\gamma_{\mathrm{L}}^{90}=\gamma \mathrm{P}$ and, as will be shown later, this assumption is not always correct.

A better approach is provided by making use of eqn. (5). Combination of this equation and eqn. (6) with the boundary condition $\gamma_{L}=\gamma_{L}$ if $\gamma_{L} \cos \theta=0$, yields the following equation:

$\gamma_{\mathrm{L}}=\gamma_{\mathrm{L}}^{\mathrm{p}}+\frac{\Delta P r_{\max }}{2 B}$

If in the above equation the values for a membrane distillation process are substituted, the calculated value of $\gamma_{L}$ is equal to $\gamma_{L}, p c$. For a proper use of eqn. (7) it is important that the value of $\Delta P$ which is substituted is higher than the maximum pressure to be applied to the system.

Experimental determination of $\gamma_{\mathrm{l}, \mathrm{pc}}^{\mathrm{p}}$

The above calculation of $\gamma_{\text {,pc }}$ reproduces reliable results. If, however, an accurate determination of $\gamma_{\mathrm{L}, \mathrm{pc}}$ is desired, the value of $\gamma \mathrm{R}, \mathrm{pc}$ has to be determined experimentally.

'This can be done by determining the 'liquid entry pressure' as a function of the surface tension of the liquid (in other words: as a function of the concentration of organic material in water). For these measurements a dry microporous membrane is put into a cell and the liquid is brought into contact with the membrane. The liquid is put under pressure and this pressure is slowly raised. The pressure at which the liquid penetrates into the membrane is defined as the liquid entry pressure. By changing the liquid composition, the liquid entry 




Fig. 2. Liquid entry pressure as a function of the welght fraction of ethanol in water for a flat PP membrane (Accurel 0.1).

pressure is obtained as a function of the concentration of organic material in water. In order to minimize the number of experiments, the calculated value of $\gamma \mathrm{L}, \mathrm{pc}$ can be used as a first estimate. In Fig. 2 the liquid entry pressure is given as a function of the weight fraction of ethanol in the mixture, using a microporous polypropylene membrane (Accurel 0.1).

Note that at the point where the liquid entry pressure is zero, the concentration is equal to the maximum allowable concentration, and the surface tension of the liquid is equal to $\gamma \mathrm{P}$.

Of course, a margin of safety should be taken. The point of operation should always be situated on the left-hand side of the curve in Fig. 2 and preferably not too close to this curve.

\section{Summary of the method}

The experimental method involves the following steps:

- determination of $\gamma \mathrm{P}$ by means of the 'penetrating drop method'

- calculation of $\gamma \mathrm{R}$,pc by means of eqn. (7), in which $\gamma_{\mathrm{L}}=\gamma \mathrm{\ell}, \mathrm{pc}$

- determination of the 'liquid entry pressure' as a function of the liquid composition (in case the calculation of $\gamma_{\mathrm{R}, \mathrm{pc}}$ is not accurate enough).

\section{Experimental}

We have seen that in this investigation two different experimental tech niques were used, namely:

- the penetrating drop method,

- the liquid entry pressure method.

Both techniques are rather simple and have already been described in the previous paragraph.

Penetrating drop method (PDM) measurements were carried out using two different kinds of polymers (PVDF and PP) with different characteristics. An overview of the characteristics of the membranes used is given in Table 1. 
TABLE 1

Properties of membranes used

\begin{tabular}{lllll}
\hline Property & Acc. 0.1 & R 5/1 & PV 159 & F 0030 \\
\hline Material & PP & PP & PVDF & PVDF \\
Configuration & flat & cap. $^{\text {a }}$ & cap. $^{\text {a }}$ & cap. $^{\text {A }}$ \\
Outer diameter $(\mu \mathrm{m})$ & - & 1800 & 1230 & 1500 \\
Inner diameter $(\mu \mathrm{m})$ & - & 1200 & 830 & 1000 \\
Membrane thickness $(\mu \mathrm{m})$ & 160 & 300 & 200 & 250 \\
Porosity $(\%)$ & ca. 80 & ca. 80 & 75 & 82 \\
Av. pore diameter $(\mu \mathrm{m})$ & 0.1 & 0.1 & & 0.60 \\
Max. pore diameter $(\mu \mathrm{m})$ & 0.40 & 0.3 & 0.25 & 0 \\
\hline
\end{tabular}

${ }^{\text {a Capillary }}$

I iquid entry pressure (I.FP) measurements were carried out on flat PP membranes and on capillary PVDF membranes. Both PDM measurements and LEP measurements were conducted with new untreated membranes which were only used in one experiment. All the membranes were kindly supplied by Fnka A.G. (Product Group Membrana).

\section{Results}

In this section the results of the penetrating drop method and the liquid entry pressure measurements will be presented. In order to be able to compare the results of these measurements with the results of measurements on homogeneous, smooth surfaces, the literature results for the latter are presented in Table 2. In this table the results of measurements on PTFE surfaces are also given.

The results of the penetrating drop method measurements are given in Table 3 , and some of these measurements are also plotted in Fig. 3 and 4. Although the values obtained for $\gamma_{\mathrm{f}}^{\mathrm{p}}$ deviate strongly from $\gamma_{\mathrm{L}}^{90}$, some interesting conclusions can be drawn from these measurements.

First, it is remarkable that the liquid penetrates into PVDF membranes at a

\section{TABLE 2}

Some properties of hydrophobic materials

\begin{tabular}{|c|c|c|c|}
\hline Property & $\mathrm{PP}$ & PVDF & PTFE \\
\hline$\gamma_{\mathrm{L}}^{90}\left(10^{-3} \mathrm{~N} / \mathrm{m}\right)$ & 55 & 50 & $40.5[6]$ \\
\hline$\gamma_{c}\left(10^{-3} \mathrm{~N} / \mathrm{m}\right)^{a}$ & $29[10]$ & $25[9]$ & $18 \quad[9]$ \\
\hline
\end{tabular}

${ }^{a} \gamma_{c}$ is the critical surface tension of wetting of a homogeneous smooth material by a liquid mixture, which is defined by the intercept of the experimental line in Fig. 1 with the line where $\theta=0\lfloor 9\rfloor$ 
TABLE 3

Penetrating drop measurements of aqueous mixtures of organic components

\begin{tabular}{|c|c|c|c|c|c|c|c|c|}
\hline \multirow{3}{*}{$\begin{array}{l}\text { Organic } \\
\text { component }\end{array}$} & \multicolumn{8}{|c|}{ Membrane } \\
\hline & \multicolumn{2}{|c|}{ PV159 } & \multicolumn{2}{|c|}{ F 0030} & \multicolumn{2}{|l|}{$\mathrm{R} 5 / 1$} & \multicolumn{2}{|c|}{ Acc. 0.1} \\
\hline & wt. $\%$ & $\gamma_{\mathrm{L}}^{\mathrm{p}}$ & wt. \% & $\gamma \mathrm{P}$ & wt. $\%$ & $\gamma_{\mathrm{L}}^{\mathrm{p}}$ & wt $\%$ & $\gamma \mathrm{p}$ \\
\hline Methanol & 37 & 37.8 & 35 & 38.5 & 69 & 29.0 & 74 & 28.0 \\
\hline Ethanol & 23.5 & 36.5 & 21 & 38.0 & 35 & 31.5 & 41 & 29.5 \\
\hline 1-Propanol & 11.5 & 33.5 & 10.5 & 35.0 & 13 & 32.0 & 15.5 & 29.5 \\
\hline 1-Butanol & 4.5 & 31.0 & 3.5 & 32.0 & 4.5 & 31.0 & 5.5 & 29.0 \\
\hline 2-Butanol & 9 & 29.0 & 7.5 & 31.0 & 8.5 & 29.5 & 9 & 29.0 \\
\hline Formic acid & 59 & 47.5 & 53 & 48.9 & 100 & - & 100 & - \\
\hline Acetic acid & 31 & 43.4 & 27 & 44.7 & 81 & 32.2 & 83 & 31.8 \\
\hline Propionic acid & 17 & 39.0 & 15.5 & 39.8 & 37 & 333 & 43 & 32.5 \\
\hline Butyric acid & 5 & 38 & 5 & 38 & 9 & 32.5 & 9 & 32.5 \\
\hline DMAC & 41 & 48 & 39 & 50 & 91 & 36 & 94 & 355 \\
\hline DMF & 43 & 48 & 39 & 50 & 95 & 36 & 98 & 355 \\
\hline DMSO & 61 & 50 & 57 & 51 & 100 & - & 100 & - \\
\hline Acetone & 33 & 35.0 & 31 & 35.8 & 47 & 31.2 & 54 & 29.8 \\
\hline 1,4-Dioxane & 37 & 44.3 & 35 & 45.0 & 61 & 38.2 & 64 & 377 \\
\hline
\end{tabular}

For PVDF as membrane material (PV 159 and $\mathrm{F} 0030) \cdot \gamma_{\mathrm{L}}^{90}=50 \times 10^{-3} \mathrm{~N} / \mathrm{m}$; for PP (R $5 / 1$ and Acc. 0 1) $\gamma_{1 .}^{90}=55 \times 10^{-3} \mathrm{~N} / \mathrm{m}$.

higher value of $\gamma_{L}$ than for penetration into PP membranes. This result is unexpected since PVDF is a more hydrophobic material than PP.

The second conclusion which can be obtained from these measurements is that all the values of $\gamma_{\mathrm{L}}^{\mathrm{p}}$ are lower than the value of $\gamma_{\mathrm{L}}^{90}$. Some of the measurements (e.g. alcohol-water mixtures in contact with flat PP membranes) give values for $\gamma \mathrm{p}$ which are almost as low as $\gamma_{\mathrm{c}}$. This means that contact angle measurements on homogeneous smooth materials form no good criterion at all for the applicability of membrane distillation. The only measurements in which values of $\gamma_{\mathrm{L}}^{\mathrm{L}}$ are roughly equal to $\gamma_{\mathrm{L}}^{90}$ are the experiments with aqueous mixtures of DMF, DMAc and DMSO on PVDF porous membranes; however, even for different types of PVDF membranes differences occur.

The third conclusion that can be drawn from the PDM experiments is that for a specific membrane $\gamma^{\mathrm{p}}$ can be dependent upon the composition of the liquid mixture. For PP membranes the value of $\gamma_{\mathrm{L}}$ seems to be dependent upon the class of organic solutes only and not on the molecular size within a series. For instance, for a flat PP membrane (Accurel 0.1) the following values for $\gamma \mathrm{L}$ are found: 
alcohols $\quad \sim 29 \times 10^{-3} \mathrm{~N} / \mathrm{m}$

carboxylic acids $\sim 32 \times 10^{-3} \mathrm{~N} / \mathrm{m}$

DMF/DMAc $\sim 36 \times 10^{-3} \mathrm{~N} / \mathrm{m}$
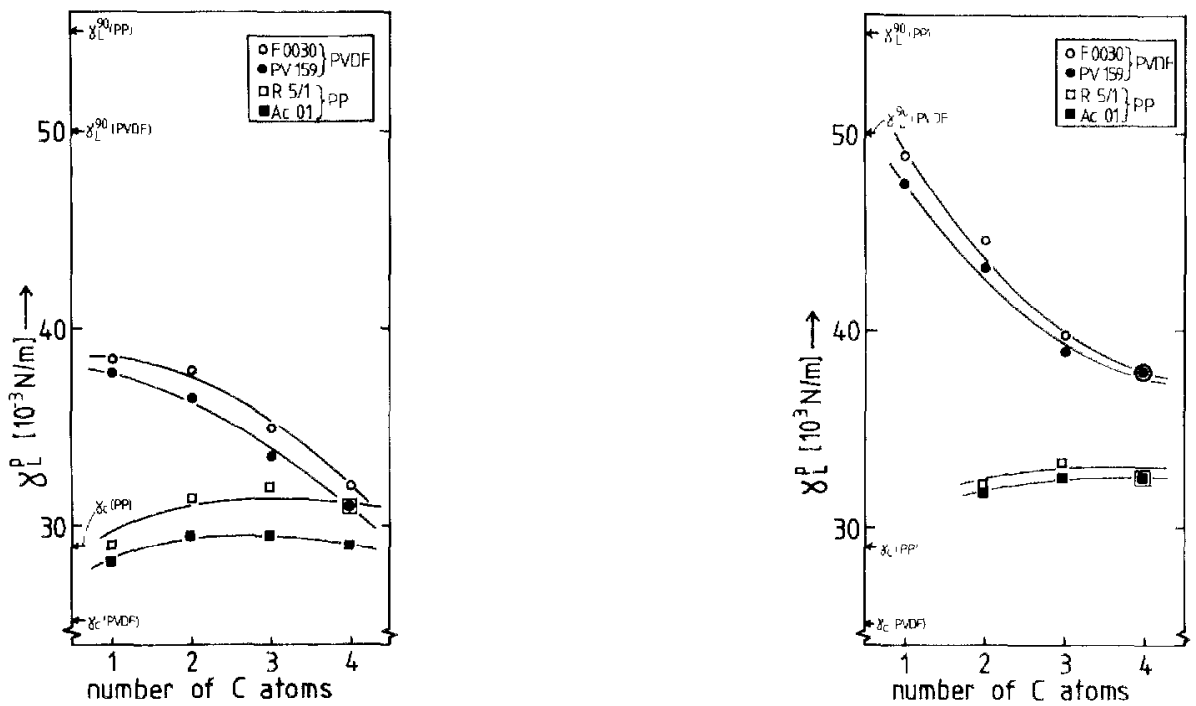

Fig. 3. $\gamma p$ for aqueous mixtures of alcohols; on the $x$-axis the number of carbon atoms of primary alcohols is plotted.

Fig. 4. $\gamma_{\text {. }}$ for aqueous mixtures of carboxylic acids; on the $x$-axis the number of carbon atoms of primary carboxylic acids is plotted.

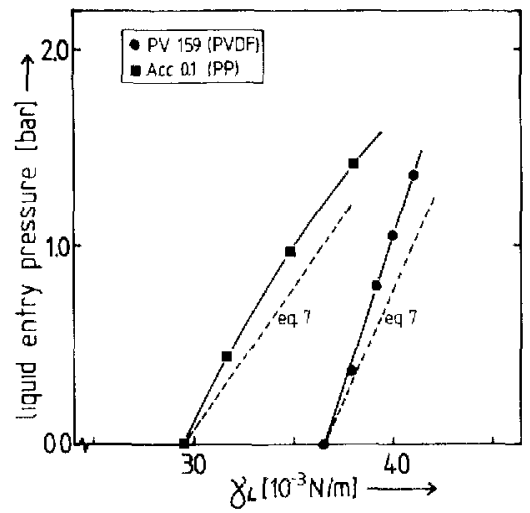

Fig. 5. Liquid entry pressure as a function of the surface tension of the liquid mixture of ethanol and water; the dashed lines are calculated by means of eqn (7) 
On the other hand, for PVDF membranes this dependence is different. Both for a series of aqueous mixtures of alcohols and for a series of aqueous mixtures of carboxylic acids the values of $\gamma \mathrm{P}$ decrease when the number of carbon atoms increases (see also Figs. 3 and 4 ).

Liquid entry pressure measurements were carried out to find out whether the values of the PDM measurements and the use of these values for the calculation of $\gamma \mathrm{E}$,pc by means of eqn. (7) are correct. The LEP measurements were carried out on flat PP membranes and on capillary PVDF membranes with aqueous mixtures of ethanol. The results of the measurements are given in Fig. 5. In this figure the LEP value is plotted as a function of the surface tension of the aqueous ethanol mixture. The values of $\gamma_{\mathrm{l}, \mathrm{pc}}$ which are calculated by means of eqn. (7) are represented in Fig. 5 by dashed lines.

From this figure it can be seen that:

- The value of $\gamma^{p}$, measured by the PDM, is in good accordance with LEP measurements.

- The values of $\gamma_{\mathrm{L}, \mathrm{pc}}^{\mathrm{p}}$ which are calculated by eqn. (7) differ from the values measured by means of the LEP. A reason for this deviation might be that $\mathrm{d}\left(\gamma_{\mathrm{L}} \cos \theta\right) / \mathrm{d} \gamma_{\mathrm{L}}$ is not exactly -1 . Furthermore, the membrane structure might be a factor of importance.

- The slope of the LEP curve is not constant. This means that the use of eqn. (7) is limited, as was already discussed before. The deviation might be caused by the fact that ethanol is not a surfactant and its mixtures with water are far from ideal.

In spite of the slight difference between the measured and the calculated curves it can be concluded that the description of the wettability criteria for a membrane distillation system by means of the penetrating drop method and the calculation of the values of $\gamma_{\mathrm{L}, \mathrm{pc}}^{\mathrm{p}}$ are rather good.

\section{Discussion}

From the results that are presented here it becomes clear that the calculation of a maximum allowable concentration of organic material on the basis of simple contact angle measurements of a droplet on a homogeneous material is not possible. The measured values of $\gamma_{\mathrm{L}}^{90}$ obtained in that way cannot be used to describe the penetration of a liquid into a porous maerial. The values of $\gamma_{\mathrm{p}}^{\mathrm{p}}$, measured by means of the PDM, are lower than $\gamma_{\mathrm{L}}^{90}$ and higher than $\gamma_{c}$, and their exact value can only be obtained by measurement.

In this paragraph a qualitative explanation for the difference between the values of $\gamma_{\mathrm{p}}^{\mathrm{p}}$ on one hand and $\gamma_{\mathrm{L}}^{90}$ and $\gamma_{\mathrm{c}}$ on the other will be given. The value of $\gamma$ e will depend upon the polymer material, the porous structure of the membrane, and the composition of the liquid (mixture). The value of $\gamma_{1}^{90}$ (and $\gamma_{c}$ ) depends upon the polymer material and in some cases also on the composition of the liquid (mixture). For instance, Bernett and Zisman [6] found different values for $\gamma_{c}$ of polyethylene when measured with different aqueous solutions 


\section{TABLE 4}

Contact angle on a smooth surface $(\theta)$ for which a porous substrate with pore fraction $f_{2}$ will be wetted $\left(\theta^{\prime}=90^{\circ}\right)$

\begin{tabular}{lllr}
\hline$f_{2}$ & $\theta\left({ }^{\circ}\right)$ & $f_{2}$ & $\theta\left({ }^{\circ}\right)$ \\
\hline 0 & 90 & 045 & 35 \\
0.2 & 75 & 0.48 & 23 \\
0.4 & 48 & 0.5 & 0 \\
\hline
\end{tabular}

$\left(\mathrm{PE}: \gamma_{\mathrm{c}}(\right.$ ethanol, 1-butanol $)=27.5 \times 10^{-3} \mathrm{~N} / \mathrm{m} ; \gamma_{\mathrm{c}}(1,4$-dioxane $)=31.5 \times 10^{-3}$ $\mathrm{N} / \mathrm{m}$ ). Although no other values for PP and PVDF are known except for the ones given in Table 2 , it is expected that $\gamma_{c}$ might be slightly different for different liquid mixtures in contact with these materials.

However, the above explanation for the possible difference in $\gamma_{\mathrm{r}}$ does not explain why the value of $\gamma_{\mathrm{L}}^{\mathrm{P}}$ is (much) lower than $\gamma_{\mathrm{L}}^{90}$. This experimental fact can be explained by the extremely high surface porosity of the membrane material. Davies and Rideal [11] stated that on rough or hairy surfaces always higher contact angles are obtained than on a smooth surface of the same material. This can be understood by the following relation, derived by Cassie and Bacter [12]:

$\cos \theta^{\prime}=f_{1} \cos \theta-f_{2}$,

which gives a relation between the contact angle measured on a rough surface $\left(\theta^{\prime}\right)$ and the contact angle measured on a smooth surface $(\theta)$. In eqn. (8) $f_{1}$ and $f_{2}$ are the fractions of the composite surface which are liquid-solid and liquid-air respectively. This equation can only be used if $f_{2}<f_{1}$. In the other case $\left(f_{2}>f_{1}\right) \cos \theta^{\prime}$ would be smaller than zero, which means that $\theta^{\prime}$ is always larger than $90^{\circ}$. This should mean that such a surface could never be wetted. However, it can be easily understood that, if $\theta$ becomes zero or approaches zero, the surface of the material (even inside the pores) becomes completely wetted.

For highly porous membranes, like the ones that are used in our investigations, the value of $f_{2}$ will be substantial and may even be larger than 0.5 , in which case eqn. (8) is no longer valid. (Note: the fact that the overall porosity of a membrane is $80 \%$ does not mean that $f_{2}$ is equal to 0.8 ; probably this value is much lower.) In cases where eqn. (8) is valid, $\theta^{\prime}$ is a function of the porosity.

In case of membrane distillation the membrane will be wetted only if $\theta^{\prime}<90^{\circ}$. For different values of $f_{2}$, the contact angles on a smooth surface, $\theta$, are listed in Table 4. The values in this table are calculated by means of eqn. (8) with the boundary condition $\cos \theta^{\prime}=0$. From this table it can be seen that for $f_{2}$ in the range of $0.45-0.5$ a small variation in porosity (or hetter: in liquid-air 
surface fraction) leads to an enormous difference in required contact angle, and will also lead to differences in $\gamma \mathrm{L}$.

It should be mentioned again that not all the results can be explained by this qualitative description. For instance, the fact that $\gamma_{\mathrm{L}}^{\mathrm{P}}$ for alcohols and carbox ylic acids on PVDF membranes is lowered as the number of carbon atoms increases, whereas the values on the PP membranes remain constant, cannot be explained by the above discussion. Therefore, further investigations, especially on the influence of membrane structure on wetting phenomena, will be carried out.

\section{Conclusions}

The main conclusion that can be drawn from our investigations is that the maximum allowable concentration of organic material in water cannot be calculated, but has to be determined experimentally.

The 'penetrating drop method' is a good and experimentally simple method for determining the maximum allowable concentration of organic material in water and its corresponding surface tension, $\gamma \mathrm{L}$.

The semi-empirical way to determine the surface tension at process conditions, $\gamma \mathrm{P}$,pc , making use of the penetrating drop method and eqn. (7) gives fairly good results, which can be used to safely estimate the maximum allowable concentration at process conditions.

\section{Acknowledgement}

This article is based upon work financially supported by the Ministerie van Economische Zaken in The Netherlands and by Enka A.G. (Product Group Membrana), Wuppertal, Federal Republic of Germany. Enka also supplied the membranes.

\section{List of symbols}

$a \quad$ activity of surfactant ( - )

$B \quad$ pore geometry coefficient $(-)$

$C \quad$ (eqn. 5) constant $(\mathrm{N} / \mathrm{m})$

$f_{1} \quad$ (eqn. 8) liquid-solid surface fraction (-)

$f_{2} \quad$ (eqn. 8) liquid-air surface fraction (-)

$\Delta P$ pressure difference $\left(\mathrm{N} / \mathrm{m}^{2}\right)$

$\Delta P_{\mathrm{v}} \quad$ vapour pressure difference $\left(\mathrm{N} / \mathrm{m}^{2}\right)$

$R \quad$ gas constant $\left(\equiv 8.310 \mathrm{~J} / \mathrm{mol}-{ }^{\circ} \mathrm{C}\right)$

$r_{\max }$ maximum pore radius $(\mu \mathrm{m})$

$\Delta T$ temperature difference $\left({ }^{\circ} \mathrm{C}\right)$

$\Gamma \quad$ surface excess activity of surfactant $\left(\mathrm{mol} / \mathrm{m}^{2}\right)$ 
$\gamma \quad$ surface tension $(\mathrm{N} / \mathrm{m})$

$\gamma_{c} \quad$ critical surface tension of wetting $(\mathrm{N} / \mathrm{m})$

$\gamma_{\mathrm{L}}^{90} \quad$ surface tension of a liquid (mixture) which has a contact angle of $90^{\circ}$ when brought in contact with a homogeneous smooth solid material $(\mathrm{N} / \mathrm{m})$

谓 surface tension of a liquid (mixture) that is on the verge of penetration into the pores of a (micro) porous membrane $(\mathrm{N} / \mathrm{m})$

$\gamma_{\mathrm{L}, \mathrm{pc}}^{\mathrm{p}} \gamma_{\mathrm{L}}^{\mathrm{p}}$ under process conditions $(\mathrm{N} / \mathrm{m})$

$\theta \quad$ contact angle $\left(^{\circ}\right)$

$\theta^{\prime} \quad$ (eqn. 8) contact angle on a rough surface $\left({ }^{\circ}\right.$ )

\author{
Subscripts \\ $i \quad$ index \\ L liquid \\ $\mathrm{S}$ solid \\ SL solid-lıquid
}

\title{
References
}

1 K. Schneider and T.J. van Gassel, Membrandestillation, Chem.-Ing.-Tech., 56 (1984) 514.

2 N. Kjellander, Applications and engineering aspects of membrane distillation, Summer School on Engineering Aspects of Membrane Processes, Aarhus, Denmark, June 2-6, 1986.

3 E.H. Lucassen-Reynders, Contact angles and adsorption on solids, J. Phys. Chem , 67 (1963) 969.

4 D. Bargeman and F. van Voorst Vader, Effect of surfactant on contact angles at nonpolar solids, J Collord Interface Sci., 42 (1973) 467.

5 H.W. Fox and W.A. Zisman, The spreading of liquids on low energy surfaces. I Polyfluoroethylene, J. Colloid Sc1, 5 (1950) 514.

6 M.K. Bernett and W.A. Zisman, Wetting of low-energy solids by aqueous solutions of highly fluorinated acids and salts, J. Phys. Chem., 63 (1959) 1241.

7 J.R. Dann, Forces involved in the adhesıve proecss. I. Critical surface tensions of polymeric solids as determined with polar liquids, J. Colloid Interface Sci., 32 (1970) 302.

8 D. Bargeman, Contact angles on nonpolar solids, J. Collord Interface Sc1., 40 (1972) 344.

9 W A. Zisman, Influence of constitution on adhesion, Ind. Eng. Chem., 55 (10) (1963) 19

10 H.Schonhorn, Heterogeneous nucleation of polymer melts on surfaces. I. Influence of sub. strates on wettability, Polym. Lett., 5 (1967) 919

11 J.T. Davies and E.K. Rideal, Interfacial Phenomena, Academic Press, New York, NY, 1963

12 A.B.D. Cassie and S Baxter, Trans. Faraday Soc., 40 (1944) 546. 